\title{
The Participle of Latin and Albanian (A Comparative Outlook)
}

\author{
Dr. Leonard Xhamani \\ Docente di Latino, Università di Tirana \\ E-mail: leonardxhamani@gmail.com
}

\section{Doi:10.5901/mjss.2014.v5n8p637}

\section{Abstract}

This article points out the characteristics of the Latin participle in comparison with the Albanian participle, a comparison which is viewed from a morphological and syntactic perspective. In both languages the participle has a double nature, that of an adjective and of a verb. Unlike Albanian, the Latin participle has two voices: the active and the passive. The active voice has two tenses: the present and the future, whereas the passive has only the perfect tense. The participle is the only non-finite form, which is formed in a syntactic way in both languages, with the relevant suffixes. Albanian has only one participle, which appears in different forms, expresses a completed action and usually has a passive meaning. With the help of the front connective article it becomes an adjective and, as such, it takes the grammatical categories of gender, number and case, following the same pattern as the Latin participle. The Latin participle is often used to show secondary circumstances during which or because of which the action in the main clause occurs. In this case, the participle replaces a subordinate clause that can express time, condition, cause, purpose, or manner. In Albanian these functions are expressed by the finite forms of the verb, which are part of the subordinate clauses of time, cause, etc. When it comes to the syntactic functions of the Albanian participle, we can say that it is used as: an attribute of a noun, separate attribute of the subject or another part of the sentence, predicative complement of the subject and the object. Though seldom, the participle has also temporal and causal functions.

Keywords: grammar, implicit forms, participle, morphology, syntax.

\section{Participio della Lingua Latina}

II participio della lingua latina, come anche quello di molte lingue indoeuropee, ha una duplice natura, quella dell'aggettivo e quella del verbo. Come aggettivo concorda nel genere, nel numero e nel caso con il sostantivo al quale si riferisce, mentre come verbo richiede il caso del sostantivo al quale si riferisce (Tantucci, 1992: 108-109). Come nella lingua albanese, anche nella lingua latina conserva lo stesso significato lessicale che ha anche il verbo dal quale deriva. Per esempio:

Homo est animal ratione utens. - Njeriu është një kafshë që përdor arsyen.

Taetra est avaritia praesertim in principibus rem publicam gubernantibus (Cic.) - E shëmtuar është koprracia veçanërisht në krerët që qeverisin një shtet.

\subsection{I tempi del participio}

I tempi del participio latino, nonché dell'infinito, hanno valore relativo, cioè non devono essere considerati separati, ma in rapporto con il verbo della frase reggente. Nella lingua latina, a differenza dall'albanese, il participio ha due diatesi: attiva e passiva. Come vedremo di seguito, per il participio della lingua albanese si può parlare solo di significati di diatesi (attiva e passiva), ma non per la diatesi come categoria grammaticale. La diatesi attiva del participio latino ha due tempi: il presente ed il futuro, mentre la diatesi passiva ha solo il passato (Tantucci: 358 )

II participio presente attivo si costruisce in maniera sintetica, tramite l'uso dei rispettivi suffissi come di seguito: al tema del presente dei verbi di prima e seconda coniugazione si aggiunge il suffisso $-n s$, mentre alla terza e quarta coniugazione il suffisso -ens. Così abbiamo:

lauda-ns (ladantis) - ai që lëvdon, duke lëvduar;

mone-ns (monentis) - ai që qorton, duke qortuar;

leg-ens (legentis) - ai që lexon, duke lexuar;

audi-ens (audientis) - ai që dëgjon, duke dëgjuar. 
His rebus conparatis Catilina nihilo minus in proxumum annum consulatum petebat sperans, si designatus foret, facile se ex voluntate Antonio usurum.

Megjithëse komploti ishte përgatitur në çdo detaj, prapëseprapë Katilina vuri kandidaturën për konsull për vitin e ardhshëm, duke shpresuar se, nëse fitonte, do ta bënte pas vetes Antonin me lehtësi (Sal. kap. XXVI).

At Pompeius cognitis his rebus, quae erant Orici atque Apolloniae gestae, Dyrrachio timens diurnis eo nocturnisque itineribus contendit...

Pompeu nga ana e tij, me të mësuar me sa kishin ndodhur në Orikum dhe në Apoloni, duke u frikësuar për Dyrrahun, u drejtua për atje, duke marshuar ditë e natë. (Caes. Liber III, kap. 13).

Come aggettivo verbale, dopo i suffissi esso prende le terminazioni della terza declinazione con un'unica terminazione (per tutt'e tre i generi). Dunque, nel caso nominativo i participi hanno rispettivamente la forma che abbiamo menzionato sopra (-ns dhe -ens).

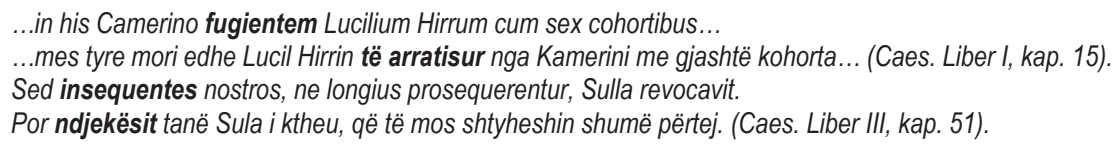

II participio presente non indica tempo, ma un'azione complementare contemporanea a quella del verbo reggente, in qualsiasi tempo sia quest'ultimo. Cioè, esprime un'azione non finita, perciò spesso si traduce con una proposizione attributiva con il pronome relativo që, seguito da un verbo all'indicativo presente, all'imperfetto 0 al futuro. Dagli esempi di sopra, però si nota che il participio si può tradurre in albanese anche con il gerundio, con aggettivo derivato dal participio oppure con il sostantivo. II participio presente non ha carattere verbale molto accentuato. La maggior parte di loro di solito vengono usati in modo definitivo come aggettivi (Basha, 1974: 34)

Nel presente, il participio latino ha sempre significato attivo (De Giorgi, P. Mandressi, 1999: 36) Questo significato hanno anche tutti i verbi transitivi e intransitivi, sia della forma attiva che quelle deponenti1: amans (transitivo attivo); veniens (intransitivo attivo); hortans (transitivo deponente); moriens (intransitivo deponente). II participio presente passivo non esiste nella lingua latina (Terracina, 2001: 56).
...si possit, inpellat ad societatem belli, existimans publice privatimque aere alieno oppressos... (existimo, are; kal) ...nëse mundte, t'u kërkonte një besëlidhje lufte, duke menduar se ata, të zhytur në hua si shtetërore dhe private...(Sal. kap. XL).
Pompeius enim discedens ab urbe in senatu dixerat eodem se habiturum loco... (discedo, ěre; jokal).
Sepse Pompeu kur po largohej nga qyteti, kishte thënë në senat se i vlerësonte njëlloj, si ata që ishin në qytet... (Caes. Liber l, kap. 33).

Dal tema del participio presente attivo in albanese sono entrate parole come: laborant - që punon; docent - që mëson (të tjerët); agjent - që vepron, ecc.

II participio passato passivo si forma dal tema del supino aggiungendo le rispettive desinenze del genitivo -us (maschile), -a (femminile) e -um (neutro), cioè si costruisce in modo sintetico. II tema del supino, per tutti i verbi, si trova togliendo la desinenza -um. Hanno il participio passato passivo:

1. Tutti i verbi transitivi, nella forma attiva. In questi casi il participio come anche nella lingua albanese ha un significato passivo. Anche la traduzione del participio passato passivo della lingua latina coincide con il participio dell'albanese oppure con l'aggettivo derivato dal participio. Per esempio:

Tema del supino
Laudat-um
Monit-um
Lect-um
Audit-um

Tema del supino

Monit-um

Audit-um

\author{
Participio passato passivo \\ laudat-us, -a, -um \\ monit-us, -a, -um \\ lect-us, -a, -um \\ audit-us, -a, -um
}

\author{
i lavdëruar, që është lavdëruar \\ i qortuar, që është qortuar \\ i lexuar, që është lexuar \\ i dëgjuar, që është dëgjuar
}

Sed re nova perturbatus maioribus itineribus Apolloniam petere coepit... (perturbo, perturbavi, perturbatum, are; kal). Por i shqetësuar nga ky lajm befasues, përpiqet të mbërrijë në Apoloni me ecje të shpejtë... (Caes. Liber III, kap. 11).

\footnotetext{
${ }^{1}$ Nella lingua latina si chiamano deponenti quei verbi che hanno perso la forma attiva e hanno conservato quella passiva, però con significato attivo. Anche questi verbi possono essere transitivi, come hortor - nxis, e intransitivi come morior - vdes.
} 
2. Tutti i verbi deponenti transitivi e intransitivi. In questi casi il participio ha un significato attivo. A questo significato nella lingua albanese corrisponde il participio passivo oppure il gerundio. Per esempio:

hortatus (deponente kalimtare = që ka nxitur); profectus (deponente jokalimtare $=$ që është nisur).

Auximo Caesar progressus omnem agrum Picenum percurrit. (progredior, progressus sum, progredi; jokal).

Duke marshuar nga Auksimi, Çezari e përshkoi të gjithë krahinën e Picenës. (Caes. Liber l, kap. 15).

Interim suos cohortatus tormenta in muris disponit certasque cuique partes ad custodiam urbis attribuit. (cohortor, cohortatus sum, cohortari; kal)

Ndërkohë, duke inkurajuar të vetët, vendos përdredhore pranë mureve dhe secilit i përcakton detyra të qarta në ruajtje të qytetit. (Caes. Liber l, kap. 17).

Come il participio dell'albanese, con l'aiuto del quale si formano le forme verbali analitiche e sintetiche, anche il participio passato passivo della lingua latina insieme alle forme personali del verbo ausiliare esse, serve per formare i tempi composti della forma passiva del modo indicativo e congiuntivo: (laudatus,a,um sum (sim); monitus,a,um eram (essem); hortatus, a,um sum (sim), ecc).

II participio futuro attivo si forma dal tema del supino, aggiungendo il suffisso - ur e le desinenze del genere degli aggettivi della prima e della seconda declinazione (-us, -a, -um).

$\begin{array}{lll}\text { Supino } & \text { Il participio futuro attivo } & \\ \text { Laudat-um } & \text { laudat-ur-us, a, um } & \text { që do të lëvdojë } \\ \text { Monit-um } & \text { monit-ur-us, a, um } & \text { që do të qortojë } \\ \text { Lect-um } & \text { lect-ur-us, a, um } & \text { që do të lexojë } \\ \text { Audit-um } & \text { audit-ur-us, a, um } & \text { që do të dëgjojë }\end{array}$

...incendia aliaque belli facinora parent: sese propediem cum magno exercitu ad urbem accessurum. ...të përgatisnin zjarret dhe mizori të tjera të luftës; dhe brenda një kohe të shkurtër do t'i afrohej qytetit me një ushtri të madhe. (Sal. kap. 32).

Sin timore defugiant, illis se oneri non futurum et per se rem publicam administraturum.

Në qoftë se shmangeshin nga frika, ai nuk do t'u bëhej barrë atyre dhe do të qeveriste vetëm me forcat e veta. (Caes.

Liber l, kap. 32).

Il participio futuro attivo indica un'azione futura in rapporto con quella del verbo reggente, cioè esprime posteriorità. Nella maggioranza dei casi, a questo participio corrisponde nella lingua albanese una forma verbale con il significato temporale del futuro.

Venio consulturus = vij për të të konsultuar (= sepse do të të konsultoj); që të të konsultoj.

Veni consulturus = erdha për të të konsultuar (= sepse do të të konsultoja); që të të konsultoja.

Veniam consulturus = do të vij për të të konsultuar (= sepse do të të konsultoj); që të të konsultoj.

In questo tempo, tutti i verbi transitivi e intransitivi, sia della forma attiva, sia di quelle deponenti, il participio ha un significato attivo, come: amaturus (kalimtare veprore = që do të dashurojë); venturus (jokalimtare veprore = që do të vijë); hortaturus (deponente kalimtare = që do të shtyjë); profecturus (deponente jokalimtare = që do të niset).

II participio futuro attivo usato da solo si vede spesso anche con il valore della proposizione finale e si può tradurre in albanese con la forma implicita del tipo për të punuar. Per esempio: (Tantucci: 359).

Darīus Cyri filiam in matrimonium recēpit, regnum firmaturus.

Dario mori për grua të bijën e Ciros, për të përforcuar mbretërinë.

\subsection{Le funzioni del participio latino}

\subsubsection{L'uso nominativo del participio}

\subsubsection{II participio nella funzione dell'aggettivo qualitativo}

II participio si può aggiungere ad un sostantivo come attributo (qualitativo), cioè per qualificare oppure determinare meglio (il participio qualitativo). Nell'albanese a questo participio di solito corrisponde un aggettivo derivato dal participio. 
Per esempio:

Item ex finitimis regionibus quas potest contrahit cohortes ex delectibus Pompeianis; in his Camerino fugientem Lucilium Hirrum cum sex cohortibus...

Prej zonave të thella, mblodhi aq kohorta sa ishte e mundur, duke bërë rekrutime të urdhëruara nga Pompeu; mes tyre mori edhe Lucil Hirrin e arratisur me gjashtë kohorta... (Caes. Liber I, kap. 15).

Incitabant praeterea corrupti civitatis mores, quos pessuma ac divorsa inter se mala, luxuria atque avaritia, vexabant.

Përveç këtyre, Katilinën e cytnin edhe zakonet e qytetit të korruptuar, mbi të cilat mbizotëronin dy vese të këqija dhe të kundërta ndërmjet tyre: luksi dhe lakmia (Sal. kap. V).

Temeritas est florentis aetatis. (Cic.)

Guximi është karakteristikë e moshës rinore.

\subsubsection{II participio nella funzione del predicato}

II participio può avere anche la funzione del predicato nominale dopo il verbo copulativo (sum) e di un complemento predicativo, specialmente con i verbi di percezione (video - shoh, sentio - ndiej, conspicio - vërej, audio - dëgjoj, ecc.) e dopo i verbi facio - bëj, induco - shtie, paraqes, fingo - formoj, gdhend ecc. (Ernout, Thomas, 1964: 282).

Multa rumor affingebat, ut paene bellum confectum videretur.

Zërat trillonin shumë sajesa, kështu që atje e shihnin pothuajse të përfunduar luftën. (Caes. Liber I, kap. 53). lugurtha iussis vestris oboediens erit.

Jugurta do t'u bindet urdhrave tuaja. (Sal. "Lufta kundër Jugurtës")

Timoleontem nemo umquam querentem audivit.

Askush nuk e dëgjoi kurrë Timoleonten të ankohej. (Cic.)

II participio passato del latino al posto di un sostantivo nella lingua albanese. - A volte, un participio passato latino si può tradurre nella lingua albanese con un sostantivo, mirando a formare un'espressione concreta: ad urbe condĭta = nga themelimi i Romës; post reges exactos = pas dëbimit të mbretërve; post urbem delētam = pas shkatërrimit të qytetit, ecc. (Tantucci: 362 ). Però si può tradurre in albanese anche con un verbo: ad urbe condíta = që kur u themelua Roma; post reges exactos = pasi u dëbuan mbretërit, ecc.

\subsubsection{L'uso verbale del participio}

Spesso il participio latino si usa per indicare le circostanze secondari, nelle quali oppure per le quali si compie l'azione della proposizione principale. Questo participio si chiama comune oppure complementare (perché è come un'apposizione che si collega al soggetto oppure un completamento della proposizione principale), e sta al posto di una proposizione subordinata. In casi simili, il participio latino ha il valore della forma del gerundio albanese.

(Vos) non potestis, voluptate omnia dirigentes (= si dirigitis), aut tuēri aut retinēre virtutem (Cic.)

Ju nuk mundni të mbroni apo të mbani virtytin, duke rregulluar (dirigentes si riferisce a vos soggetto della frase principale) çdo gjë sipas qejit.

A differenza dall'albanese, dove il participio nella maggioranza dei casi ha una funzione attributiva, il participio latino ha funzioni numerose, le quali mancano nella nostra lingua. Come vedremo di seguito, il participio comune latino può avere nella proposizione una funzione temporale, causale, concessiva ecc. Nella lingua albanese, queste funzioni si esprimono con forme implicite del verbo, le quali sono parte della proposizione subordinata temporale, causale, concessiva ecc., oltre alla funzione modale, la quale corrisponde meglio al gerundio dell'albanese. Per esempio :

\subsubsection{1 con funzione temporale:}

Pompeius enim discedens (= dum discedit) ab urbe in senatu dixerat eodem se habiturum loco...

Sepse Pompeu, kur po largohej nga qyteti, kishte thënë në senat se i vlerësonte njëlloj, si ata që ishin në qytet...

(Caes. Liber I, kap. 33).

Cornelius Rufus dormiens (= dum dormit) oculorum visus amisit (Plin.).

Kornel Rufi humbi dritën e syve, ndërsa flinte. 


\subsubsection{2 con funzione causale:}

Ita illi ianua prohibiti tantum facinus frustra susceperant.

Kështu, plani i kobshëm u shkoi kot komplotistëve, sepse u penguan që te pragu. (Sal. kap. XXIX).

Dionysius cultros metuens (= quia metuebat) tonsorios, candenti carbone sibi adurebat capillum (Cic.).

Dionizi, ngaqë kishte frikë briskun e rrojes, i digjte qimet e mjekrës me një karbon të ndezur.

\subsubsection{3 con funzione concessiva:}

Quoniam quidem circumventus ab inimicis praeceps agor, incendium meum ruina restinguam.

Edhe pse jam i rrethuar prej armiqve që më shtyjnë drejt humbjes, zjarrin tim do ta shuaj me shkatërrimin e shtetit.

(Sal. kap. XXXI).

Pueri Spartiatae non ingemiscunt verběrum dolore laniati.

Fëmijët spartanë nuk qajnë, edhe pse brehen nga dhimbja e kamxhikut. (Cic.)

\subsubsection{4 con funzione condizionale:}

Ni virtus fidesque vostra spectata mihi forent, nequiquam opportuna res cecidisset.

Sikur të mos shihja guximin dhe besnikërinë tuaj, do të mendoja se më kot na është paraqitur ky rast i favorshëm. (Sal. kap. $X X)$.

Ne mente quidem recte uti possŭmus multo cibo et potione completi (= si completi sumus).

Nuk mund të përdorim mirë as mendjen, nëse jemi të rënduar nga shumë ushqim dhe pije. (Cic.)

\subsubsection{5 con funzione finale:}

Hoc idem Caesar facere cogebatur, ut submissis in eundem locum cohortibus defessos reciperet.

Çezari ishte $i$ detyruar të bënte të njëjtën gjë, me qëllim që të dërgonte kohortat e freskëta në atë pozicion dhe tërhiqte të lodhurit. (Caes. Liber I, kap. 45).

Hannibal in Etruriam ducit, eam quoque gentem aut vi aut voluntate adiuncturus (= ut adiungěret).

Hanibali udhëhoqi ushtrinë në Etruria, për të bashkuar me forcë apo në mënyrë të pavullnetshme edhe atë popull.

\subsubsection{6 con funzione modale:}

Ad hoc maledictis increpabat omnis bonos, suorum unumquemque nominans laudare...

Pas këtyre filloi të shpifte e të mallkonte qytetarët më të ndershëm, ndërsa lëvdonte njerëzit e vet, duke i thirrur një nga një. (Sal. kap. XXI).

Consul Lentulum, quod praetor erat, ipse manu tenens in senatum perducit...

Konsulli e shoqëroi në senat Lentulin personalisht, duke e mbajtur për dore, sepse ishte pretor... (Sal. kap. XLVI).

In rare occasioni con funzione temporale e causale si usa anche il participio della lingua albanese.

\section{II Participio della Lingua Albanese}

Con il termine participio (latino participium) viene chiamata quella forma implicita che ha caratteristiche semantiche e grammaticali dei verbi, anche aggettivali (Instituti i Gjuhësisë dhe i Letërsisë, 1995: 331). Dai grammatici antichi è stato definito così perché prende sia dalle caratteristiche del sostantivo (concretamente dall'aggettivo), sia dalle caratteristiche del verbo. Perciò, nelle lingue antiche, il participio ha avuto terminazioni a seconda del genere e del numero e veniva declinato come i sostantivi e gli aggettivi (lat. laudatus - laudata - laudatum). II participio latino ha forme particolari addirittura per la forma attiva e passiva.

Per quanto riguarda l'albanese, essa ha solo un participio che appartiene al passato e generalmente ha un carattere passivo. II tema verbale, dal quale si forma il participio, può coincidere con quello del passato remoto. Questo è naturale, visto che il participio dell'albanese è un participio passato, coincidendo il suo significato temporale con quello del passato remoto. II tema verbale, però, dal quale si forma il participio può coincidere anche con quello del presente oppure può essere diverso dai temi di questi tempi (Instituti i Gjuhësisë dhe i Letërsisë: 299). 


\subsection{Come si forma il participio in albanese}

1. Formano il participio da un tema verbale che coincide con quello del passato remoto (Demiraj, 1986: 936):

a. Tutti i verbi della prima coniugazione. II participio dei verbi con il tema in -0-, -ua-, rispettivamente -e-, ye- esce con il dittongo delle vocali -ua-, rispettivamente -ye-, così come nel passato remoto plurale: p.es. la-va - larë; fshi-va - fshirë; punua-m - punuar; lexua-m - lexuar; shkrua-m - shkruar; përkthye-m përkthyer; lye-m - lyer; gjet-a - gjetur; mbajt-a - mbajtur; bër-a - bërë.

b. I verbi della seconda coniugazione oltre a quelli che cambiano -e- / -je-, -o- nel passato remoto: p.es. hap-a - hapur; njoh-a - njohur; fol-a - folur; thirr-a - thirrur; vra-va - vrarë ecc.

c. I verbi della terza coniugazione: di, fle, ngre e pi. Così abbiamo: dit-a - ditur; fjet-a - fjetur; ngrit-a ngritur, ecc.

d. I verbi irregolari quali: jam, rri, shoh ecc. Così abbiamo: qe-shë - qenë; ndenj-a - ndenjur; pa-shë - parë.

2. Formano il participio da un tema verbale che coincide con quello del presente:

a. Alcuni verbi della seconda coniugazione quali: heq (hoqa) - heq-ur; pjek (poqa) - pjek-ur; vjel (vola) vjel-ë; nxjerr (nxora) - nxjerr-ë; dal (dola) - dal-ë, sjell (solla) - sjellë. (Agalliu, 1983: 45)

b. Alcuni verbi della terza coniugazione quali: vë, zë, përzë, nxë come anche il verbo irregolare lë: vë (vura) - vë-në; zë (zura) - zë-në; përzë (përzura) - përzë-në; nxë (nxura) - nxë-në; lë (lashë) - lë-në.

3. Formano il participio da un tema verbale che è diverso da quello che passato remoto e del presente, i verbi: bie (ra-shë) - rë-në; ha (hëngr-a) - ngrënë; jap (dha-shë) - dhë-në; dua (desh-a) - dashur; them (thash-ë) thë-në; vdes (vdiq-a) - vdek-ur ecc.

Dagli esempi di cui sopra si può facilmente notare che i suffissi con cui si forma il participio sono: -rë/-r, -ur, -ë dhe -në. Dunque, l'albanese ha soltanto un participio che si rileva con forme diverse. Questi suffissi si scontrano nell'opera di Buzuku (Lafe, 1962: 154). Da tutte le forme implicite dell'albanese, soltanto il participio è costruito in modo sintetico, così come il participio latino, con suffissi che abbiamo menzionato sopra, i quali sono ereditati da una prima fase indoeuropea. Comunque, alcuni tipi di participi indoeuropei non sono stati conservati. Uno di questi si pensa sia il participio con -nt, il quale ha un significato attivo ed è conservato anche nel latino (laudans, laudant-is; amans, amant-is). Definivamente l'albanese ha perso il participio antico indeuropeo del tipo con -nt. Con la funzione di questo participio spesso si usa il gerundio (duke punuar).

Nella lingua albanese il participio è una forma verbale solidificata che si trasforma in aggettivo con l'aiuto dell'articolo. Esso, a differenza del participio latino, non ha nessuna dalle categorie grammaticali che la caratterizzino come forma verbale con caratteristiche del verbo e dell'aggettivo (diatesi, tempo, genere e numero) (Topalli, 2010: 448). Si parla delle categorie grammaticali di solito solo quando ai significati grammaticali corrispondono anche forme grammaticali differenti. Da questo punto di vista il participio si presenta invariabile. II participio albanese non ha il tempo come categoria grammaticale, perché manca l'opposizione formale, ma ha il tempo come significato grammaticale, visto che questo significato è caratteristica del verbo come classe di parole, entro le quali si comprende anche il participio. Questo si nota anche nel participio latino, il quale non indica un tempo particolare, però indica un'azione complementare contemporanea a quella del verbo reggente, in qualsiasi tempo sia quest'ultimo, cioè, esprime un'azione non ancora finita. Per quanto riguarda la categoria grammaticale della diatesi, la quale nella lingua albanese si nota chiaramente tramite i mezzi morfologici di qualsiasi modo e forma temporale, occore dire che anch'essa manca al participio. Per il participio si può parlare solo di significati di diatesi (attiva, passiva) e non della diatesi come categoria grammaticale, perché il participio non ha forme per esprimere questi significati, cioè, non come il participio latino, nel quale si nota bene la diatesi. Nei verbi transitivi, usato come membro a parte, il participio ha un significato passivo. P.es:

Gjermanët e ngurosur, shtrirë të vdekur në sallon, dhe vajza e doktorit, që kujtonte se i kishte helmuar,... Rojtari i natës $i$ Institutit të Higjenës, e kishte nxjerrë një këngë për kujtim të të dashurës së tij, shtypur prej makinës-cisternë të ujrave të zeza,... (Kadare, Darka e gabuar, f. 72, 107).

Questo si dimostra anche dal fatto che gli aggettivi articolati formati dal tema del participio di quei verbi in generale hanno significato passivo: i vrarë, i zbukuruar, i hapur, i mbyllur, i përkthyer ecc. Per esempio:

Një copë herë nuk e gjente dot se ç’kohë ishte dhe përse ndodhej në dhomën e prindërve, e shtrirë, siç kishte rënë, e veshur, mbi shtrat. (Kadare, Darka e gabuar, f. 68).

Një mburrje e re, e shfaqur, me sa dukej, për herë të parë në botë, dëgjohej gjithandej,... (Kadare, Spiritus, f. 82).

Ma il participio di alcuni verbi transitivi si usa anche con significato attivo. Questo viene dimostrato anche dagli 
aggettivi derivato dal participio sostantivati, quali: i ditur (ai që di), i pirë (ai që ka pirë), i gjezdisur (ai që gjezdis) ecc. (Demiraj, 1976: 179). II participio dei verbi intransitivi ha significato attivo (intransitivo): $i$ shëtitur, $i$ ardhur, i fjetur, i ndenjur ecc. (Instituti i Gjuhësisë dhe i Letërsisë: 331). Anche nella lingua latina, come abbiamo visto sopra, il participio passato di alcuni verbi quali: potus (poto) = që ka pirë; cenatus (ceno) = që ka drekuar; pransus (pranděo) = që ka ngrënë, ecc. ed il participio futuro di tutti i verbi transitivi e intransitivi, sia della forma passiva sia di quelle depontenti, ha significato attivo. Per esempio:

\begin{abstract}
...dhe ai, sekretar i parë i rinisë së krejt Universitetit, $i$ ardhur për mbikëqyrje nga Tirana, me këmishë ngjyrë qielli,... Tmerri i verbër i pashkak, i ardhur nga një kah i pamundur, e rrënqethi prapë. (Kadare, Spiritus, f. 82, 84).

II participio è limitato nei significati, nella forma grammaticale e negli usi. Dunque, esso non ha nessuna categoria grammaticale verbale e non verbale. Ciò nonostante, il participio deve essere compreso nel sistema verbale dell'albanese per queste ragioni:
\end{abstract}

1. Esso conserva generalmente il significato verbale dell'azione o dello stato. Quando si usa come termine a parte, può reggere complementi e membri che indicano l'agente (përfolur nga bota, mallkuar nga njerëzit). Come le forme implicite, il participio esprime un'azione come caratteristica (Agalliu, 1972: 55) e non come processo. (Çeliku, 2006: 74).

2. Con il suo aiuto si formano le forme verbali analitiche e sintetiche. Esso serve per formare i tempi composti del passato, del presente e dell'imperfetto del modo ammirativo (Instituti i Gjuhësisë dhe i Letërsisë: 291) ecc. Questo si nota anche nel participio passato passivo della lingua latina, il quale insieme alle forme attive del verbo ausiliare esse, serve a formare i tempi composti della forma passiva del modo indicativo e congiuntivo.

3. Esso si usa con valore verbale dopo i verbi modali do dhe duhet (Instituti i Gjuhësisë dhe i Letërsisë: 263)

Queste sono alcune caratteristiche limitate verbali del participio che ci costringe a includerlo nella classe dei verbi e non chiamarlo aggettivo. Anche se ha numerose funzioni sintattiche esso non costituisce una parte del discorso, ma una parte del paradigma della flessione del verbo (Memishaj, 2007: 61). Comunque nel participio della forma punuar si intrecciano le caratteristiche limitate verbali e molto limitate aggettivali. Basandosi in questo intreccio di caratteristiche, riteniamo che non bisogna tenere una posizione definitiva per collegare il participio soltanto con il verbo oppure soltanto con l'aggettivo.

II participio della lingua albanese indica un'azione compiuta e ha principalmente un significato passivo. Nella maggioranza dei casi il participio separato può essere preso per un aggettivo derivato dal participio. In casi simili si tratta di due forme facoltative dell'albanese standard. Per esempio:

Po rrinte në ballkon, ngjeshur pas murit (ose i ngjeshur), duke përgjuar lëvizjet në rrugë. Brenda fashikujve zbulova një vjershë, shkruar (ose të shkruar) mbi një fletë fletoreje. (Kongoli, Jetë në një kuti shkrepësesh f. 23, 36).

Nella lingua letteraria albanese si usa di più l'aggettivo derivato dal participio, assumendosi i rispettivi indicatori morfologici. Con l'aiuto dell'articolo, grammaticalmente, da qualsiasi participio si può formare un aggetivo, ma non sempre è stato arrivato alla cristallizzazione di una nuova unità lessicale, di un aggettivo per eccellenza. Diventano aggettivi i participi di quei verbi, i quali hanno una grande frequenza d'uso ed una ricca struttura semantica. Un participio articolato diventa un aggettivo quando perde il significato dell'azione ed acquisisce un nuovo significato; quando prende la categoria del grado e quando l'articolo diventa presente in tutte le funzioni. Quindi, che un participio articolato diventi un aggettivo, deve adempiere a questi criteri: cambiamento semantico, categoria del grado e la flessione dell'articolo. L'articolo negli aggettivi derivati dai participi serve anche per formare nuove parole, anche come indicatore della categorie della declinazione, genere e numero, ha cioè una duplice funzione. L'articolo, cioè, è un elemento grammaticale che acquisisce capacità formative, transformando le caratteristiche morfologiche del participio (Instituti i Gjuhësisë dhe i Letërsisë: 69). Comunque sia questa rimane una questione delicata che bisogna studiarla passo dopo passo con il passar del tempo. Portiamo un esempio per capirlo meglio: Dheu $i$ hedhur nga makina kishte zënë rrugën. Formalmente qui abbiamo un aggettivo articolato, ma nel campo lessicale non abbiamo un aggettivo per eccellenza, poiché $i$ hedhur continua ad indicare un'azione, che è una caratteristica del verbo; l'articolo non è parte permanente della struttura della parola (esso può anche non essere espresso: Dheu, hedhur nga makina, kishte zënë rrugën); non ha i gradi degli aggettivi qualificativi; $i$ hedhur ha le caratteristiche sintattiche del verbo, cioè si può accompagnare dal complemento d'agente (i hedhur nga makina, dje, në rrugë) (Thomai, 2006: 181). Se avessimo: Është djalë $i$ hedhur ai qui abbiamo un aggettivo per eccellenza. L'articolazione del participio è arrivato come risultato della sua unione con la parola determinata. In casi simili dobbiamo stare attenti all'interpretazione dei suoi valori semantici. Dobbiamo tener presente che il participio anche se ha assunto la forma di un aggettivo articolato, dal punto di vista semantico non è 
passato nella categoria degli aggettivi (Demiraj, 1970: 84).

\subsection{Le funzioni sintattiche del participio}

Il participio della lingua albanese ha alcune funzioni. Esso può essere nella frase:

I. Attributo di un sostantivo, che lo determina in modo diretto. In quanto tale, il participio segue il sostantivo e non viene separato dalla virgola. In questa funzione esso viene accompagnato quasi sempre da un altro membro determinativo con rapporti oggettivi, complementari ecc. (Agalliu: 61). Per esempio:

Eci përmes kopshtit rrethuar me pemë të larta. Njohu rrobat e të vëllait grisur gjatë rrugës.

I përkiste fqinjës së apartamentit të katit të parë, në shkallën ngjitur me ne. (Kongoli, Jetë në një kuti shkrepësesh, f. 93).

II. Attributo separato del soggetto oppure di un altro membro della frase, espresso con sostantivo o pronome. Possiamo dire che questo è uno degli usi più principali del participio ed il principale per la forma senza articolo. In questa funzione si usano sia i participi dei verbi transitivi che quelli dei verbi intransitivi. In casi simili, il participio viene direttamente dopo la parola determinata (Çeliku: 199). Per esempio:

Babai, shtrirë në divanin e tij, dëgjonte me qetësi lajmet. Ajo kishte veshur një triko të kuqe, punuar disa vite më parë. Njerëzit, mbledhur rreth zjarrit, diskutonin për motin e keq.

Prej andej mund të kontrolloje platenë, domethënë pjesën kryesore të oborrit të vilës, mbushur me tryeza pranë njëratjetrës. Praninë e tij e vunë re edhe banorë të një pallati pesëkatësh, ndërtuar rishtas me punë vullnetare. (Kongoli, Jetë në një kuti shkrepësesh, f. 77, 96).

III. Attributo predicativo del soggetto e del complemento (Paço, 1981: 84). In una funzione simile, il participio si usa nelle costruzioni nome (pronome) nel caso nominativo + il participio e nome (pronome) nel caso accusativo + il participio. Tali costruzioni si trovano vicino ai verbi espliciti determinati che indicano solitamente stato: rri, qëndroj, mbes, lë, mbaj, kam ecc.

Atë natë drita qëndroi ndezur gjer në mëngjes.

Ajo tërë jetën kishte jetuar zhveshur e zbathur. E kishte kokën ulur.

Me rrëmbim mori pallton e dimrit hedhur sipër karriges. (Kadare, Darka e gabuar, f. 172).

...një tryezë mbi të cilën gjendej kompiuteri, ca aparatura të tjera, një karrige veshur me lëkurë të zezë, e rrotullueshme

dhe, si kudo, portreti i biondes me syze. (Kongoli, Jetë në një kuti shkrepësesh, f. 45).

L'interpretazione come predicato nominale non lascia ombra di dubbio, quando abbiamo a che fare con il participio dei verbi intransitivi, i quali non hanno diatesi passiva-riflessiva, di conseguenza nemmeno forma passiva del perfetto, costruita con l'ausiliare jam.

Ata nuk e dinë në jemi gjallë a vdekur.

Sikur ndërtesa me tulla të kuqe e kishte thithur dhe ajo nuk kishte ecur, por fluturuar drejt saj. (Kadare, Spiritus, f. 165).

II participio del verbo shkoj-shkuar con sostantivi in funzione predicativa (attributo predicativo oppure predicato nominale) e con numerali cardinali ha assunto il valore di un avverbio di quantità al grado comparativo (Agalliu: 63).

Ky është flori e shkuar floririt.

Kjo ishte e natyrshme, përderisa në krye të tyre kishte dy a tre shefa serbë, për të cilët fjalët "Shqipëri etnike" ishin mortje e shkuar mortjes. Por koloneli vazhdoi me të njëjtin zë: një mik të madh, një shok universiteti, më të ngushtin e miqve, vëlla e shkuar vëllait. (Kadare, Darka e gabuar, f. 20, 41).

Nella funzione dell'attributo del soggetto oppure del complemento, il participio può presentarsi anche come membro allargato, ma anche no. II participio come membro allargato, di solito con un'apposizione di luogo, modo, causa, ha il valore di un verbo, mentre non allargato in molti casi tende all'avverbio. Nella lingua albanese un gruppo non esiguo di avverbi ottenuti dai participi con conversione. Tali possiamo menzionare: shtruar, hidhur, kaluar prerë, shkoqur, mbytur ecc. II numero dei participi usati come avverbi sta aumentando 
(Hysa, 1970: 49). Se i participi non indicano un'azione come caratteristica di un oggetto e indicano la modalità del compimento dell'azione, quando non vengono sostituiti dal participio articolato e quando acquisiscono la categoria del grado, di conseguenza si usano nelle frasi come complemento di modo e questo dimostra che sono passati definitivamente alla classe degli avverbi. Anche nella lingua latina il participio si usa nella funzione modale, ma non trasformandosi in un avverbio. Per esempio:

Ai buzëqeshi hidhur..., ai po fliste shtruar... Dje foli më prerë... etj.

Ta marrim shtruar, - tha sekretari i dytë. Nervozizmi i tij dukej hapur, por tani aq i bënte. (Kadare, E penguara, f. 17, 58).

IV. II participio si usa anche in una costruzione a parte come attributo predicativo di un sostantivo preceduto dalla preposizione me, il quale gioca il ruolo di un membro secondario della frase:

Atë e zuri gjumi me kokën mbështetur në mur. Në shkollë erdhi me krahun e djathtë fashuar deri në bërryl.

V. A. Xhuvani ha messo in risalto una delle funzioni più frequenti del participio, quella temporale (Xhuvani, 1980: 252), in frasi simili:

Gjendur rastësisht në breg të lumit...

Sono rare anche le costruzioni con valore causale, funzione che scontriamo anche nel participio latino. Per esempio:

Mbështjellë me mushama, ata nuk njiheshin fare².

Nei nostri giorni tali usi del participio si scontrano frequentemente, specialmente nello stile politico-scientifico, quali: nisur nga kushtet, bazuar në ligj, mbështetur në nenin..., parë nga ky këndvështrim ecc. Questi participi possono essere usati al posto del gerundio, con il quale si possono facilmente scambiare:

Nisur (duke u nisur) nga situata aktuale, ndryshimet duhen bërë sa më parë.

Lidhur (duke e lidhur) me atë që folëm në Komitet të Partisë, s'kam gjetur asgjë të re. (Kadare, E penguara, f. 48).

Comunque, possiamo affermare che nella lingua scritta si evitano queste costruzioni, il che si spiega con l'aumento dell'uso dell'aggettivo verbale che conserva il significato del participio.

VI. L'uso del participio dopo i verbi modali duhet e do. II verbo duhet esprime il legame con il soggetto, mentre il participio del verbo esprime il contenuto lessicale.

Duhen ndërruar tubacionet, thonin punëtorët e komunales. (Kongoli, Jetë në një kuti shkrepësesh, f. 164).

Si shumë nga ata që kishin studiuar në Gjermani, kishte pasur një prije, një joshje, por kjo s'duhej ngatërruar me nazizmin. (Kadare, Darka e gabuar, f. 161).

Kjo punë duhet biseduar me ministrin. Është një rast i rrallë që s'duhet humbur. (Kadare, Spiritus, f. 104).

Nderi do shpërblyer me nder.

Do pyetur edhe ai për këtë punë.

In costruzioni simili si usa solo il participio articolato, il cui significato differisce da quello abituale, cioè non indica un'azione usata nel passato, ma un'azione nel significato della possibilità o della necessità. Questo succede a causa dei verbi modali e dal punto di vista della funzione è equivalente all'infinito (Domi, 1961: 67). e può essere sostituito da esso (si tratta dell'infinito del tipo me ba): Duhet shkue atje - duhet me shkue atje.

Questo è giustamente rifiutato da Sh. Demiraj, poiché in questo tipo di sintagma il secondo membro, quando il verbo è transitivo, ha un significato passivo, così come il participio passato (Demiraj, 1969: 89). Dunque, anche se il participio preceduto dai verbi modali si può sostituire dall'infinito, esso si interpreterà come participio, poiché in nessuno dei casi non compie le funzioni caratteristiche dell'infinito.

\footnotetext{
2 Un caso simile, M. Çeliku lo riconosce come "participio nella funzione del determinativo separato, dove le relazioni determinative si aggiungono alle relazioni circostanziali secondarie", opera citata, p. 197.
} 


\section{Le Conclusioni}

Il participio è l'unica forma implicita, la quale, nella lingua latina, come anche in albanese, si costruisce in maniera sintetica, con i rispettivi suffissi. II participio in queste lingue ha una duplice natura, quella dell'aggettico e quella del verbo. Come aggettivo il participio latino concorda in genere, numero e caso con l'aggettivo al quale si riferisce, mentre come verbo richiede il caso del sostantivo al quale si riferisce. II participio ha due diatesi: attiva e passiva. La diatesi attiva ha due tempi: il presente ed il futuro, mentre la diatesi passiva ha solo il passato. Tutt'e tre i tempi del participio latino si costruiscono in modo sintetico, tramite i rispettivi suffissi.

II participio della lingua albanese invece indica un'azione compiuta ed ha principalmente significato passivo. L'albanese non ha un particpio presente e nella forma in cui appare, è limitato in significati, forme grammaticali ed usi. II participio si trasforma in aggettivo grazie all'articolo. In casi simili, esso concorda con il sostantivo come il participio latino in genere, numero e caso; esso formalmente coincide con l'aggettivo, mentre semanticamente coincide con il participio. Nella lingua letteraria albanese si usa di più l'aggettivo derivato dal participio.

II participio latino è molto più ricco per quanto riguarda le categorie grammaticali. Esso ha tre tempi: II participio presente, il quale spesso si traduce in albanese con frasi attributive con il pronome relativo që, seguito da un verbo del modo indicativo, però si può tradurre anche con il gerundio, con l'aggettivo derivato dal participio oppure con un sostantivo. Nel presente il participio latino ha sempre significato attivo.

II participio passato passivo il quale, come il participio albanese, ha un significato passivo. Questo participio latino, nella maggioranza dei casi viene dato in albanese con il participio oppure con l'aggettivo derivato dal participio, qualche volta anche con il gerundio.

II participio futuro attivo, al quale in albanese, nella maggioranza dei casi, corrisponde una forma verbale con il significato temporale del futuro. II participio futuro attivo usato separato si vede spesso anche con il valore della proposizione finale e si può tradurre in albanese con la forma implicita del tipo për të bërë.

Il participio latino si può usare anche nella funzione dell'aggettivo qualificativo, aggiungendosi ad un sostantivo per qualificarlo o determinarlo meglio. In albanese, a questo participio, corrisponde di solito un aggettivo derivato da un participio, nella funzione di un attributo. II participio della lingua latina, però, lo troviamo anche da solo con la funzione di un sostantivo.

Spesso il participio latino si usa per indicare le circostanze secondarie, nelle quali oppure per le quali si compie l'azione della frase principale. In questo caso esso rimane nel posto di una frase dipendente, la quale può avere funzioni che il participio dell'albanese non ha, quali: temporale, concessiva, finale e modale. Nella lingua albanese, queste funzioni si esprimono con forme implicile del verbo, le quali sono una parte della frase subordinata temporale, causale, concessiva ecc. tranne la funzione modale, la quale corrisponde meglio al gerundio dell'albanese. Comunque, a partire dagli esempi, raramente il participio dell'albanese si usa con funzione temporale e causale.

Per quanto riguarda le funzioni sintattiche del participio dell'albanese, possiamo dire che esso si usa come: attributo di un sostantivo, che lo determina direttamente; attributo separato del soggetto oppure di un altro membro della frase, espresso con sostantivo oppure con pronome; attributo predicativo del soggetto e del complemento oggetto; come attributo predicativo del sostantivo preceduto dalla proposizione me. Cioè, la sua funzione principale è quella dell'attributo.

II participio passato passivo della lingua latina insieme alle forme personali del verbo ausiliare esse, serve per formare i tempi composti della forma passiva, del modo indicativo e congiuntivo. Questo si nota nella lingua albanese, dove il participio insieme al verbo ausiliare kam e jam, serve per formare i tempi composti del verbo.

\section{References}

Agalliu Fatmir (1972), Pjesorja në gjuhën shqipe, SF, 2, Tiranë.

Agalliu Fatmir (1983), Ç'është tema e eptimit?, Gjuha jonë, 2, Tiranë.

Akademia e Shkencave, Instituti i Gjuhësisë dhe i Letërsisë (1995), Gramatika e gjuhës shqipe, I, Tiranë.

Basha Nermin (1974), Përcjellorja e shqipes dhe gerundi e participi i latinishtes, punim diplome, Tiranë.

Caesar Caius Julius (2008), De bello civili, Torino: UTET.

Çeliku Mehmet (2006), Format e pashtjelluara të foljes në gjuhën e sotme shqipe, Tiranë: Shblu.

De Giorgi Marco, Mandressi Piero (1999), I verbi latini, Milano: Hoepli.

Demiraj Shaban (1969), Rreth disa problemeve të paskajores në gjuhën shqipe, SF, 1, Tiranë.

Demiraj Shaban (1976), Morfologji historike e gjuhës shqipe, (pjesa II), Tiranë: Mihal Duri.

Demiraj Shaban (1986), Gramatikë historike e gjuhës shqipe, Tiranë: "8 Nëntori".

Domi Mahir (1961), Morfologjia historike e shqipes, konspekt leksionesh, Tiranë. 
Ernous Alfred, Thomas François (1964), Syntaxe latine, Paris: Klincksieck.

Famerie Etienne, Bodson Arthur, Dubuisson Michel (2012), Methode de langue latine, Paris: Armand Colin.

Hysa Enver (1970), Ndajfoljet në gjuhën e sotme shqipe, Studime Filologjike, 3, Tiranë.

Kadare Ismail (1996), Spiritus, Tiranë: Onufri.

Kadare Ismail (2008), Darka e gabuar, Tiranë: Onufri.

Kadare Ismail (2009), E penguara, Tiranë: Onufri.

Kongoli Fatos (2009), Jetë në një kuti shkrepësesh, Tiranë: Toena.

Lafe Emil (1962), Trajtat e pjesores te Gjon Buzuku, Konferenca I e studimeve albanologjike, Tiranë.

McKeown Jc., 2010. Classical Latin, Indianapolis: Hackett Publishing.

Memishaj Valter (2007), Rreth mbiemrave prejpjesorë në shqipe dhe në greqishte, SF, 3-4, Tiranë.

Paço Eliana (1981), Pjesorja në veprën e P. Bogdanit, SF, 3, Tiranë.

Piazzino C., Fragonara A. (1985), Humanitatis magistra, Torino: Paravia.

Sallustius Caius Crispus, (2008), Catilinae coniuratio, Milano: Oscarmondadori.

Tantucci Vittorio (1992), Urbis et orbis lingua, Bologna: Poseidonia.

Terracina Francesco (2003), Verbi latini, Tutti i verbi regolari e irregolari, Milano: Vallardi.

Thomai Jani (2006), Leksikologjia e gjuhës shqipe, Tiranë: Toena.

Topalli Kolec (2010), Sistemi foljor i gjuhës shqipe, Tiranë: Plejad.

Xhuvani Aleksandër (1980), Pjesorja në shqipet; Paskajorja në shqipet, Vepra I, Tiranë: Kombinati Poligrafik. 